\title{
Comparison of Set-membership and Interval Observer Approaches for State Estimation of Uncertain Systems
}

\author{
Masoud Pourasghar ${ }^{1,2}$, Vicenç Puig ${ }^{1,2}$ and Carlos Ocampo-Martinez ${ }^{1,2}$
}

\begin{abstract}
This paper presents the analysis and comparison of interval-observer-based and set-membership approaches for the state estimation of linear uncertain systems. In both approaches noise and disturbances are assumed to be unknown but bounded. In this paper, both approaches are compared when implemented using zonotopes. Mathematical expressions of both approaches are compared and conditions under which they provide the same state estimation are derived. At the end, an example based on a two-tanks system is used to test the obtained conditions.
\end{abstract}

\section{INTRODUCTION}

The state estimation problem is one of the significant problems in control theory. There are various approaches in the literature for state estimation. These approaches can be classified in two main categories: stochastic and deterministic. According to [1], different ways to model the noises and perturbations are the main difference between stochastic and deterministic approaches. In stochastic approaches, noises and perturbations are assumed to be described by some known statistical distribution (typically Gaussian) but deterministic approaches consider noises and disturbances as unknown variables with known bounds. Inside the family of deterministic approaches, the interval state observers and set-membership state estimators have been introduced separately [2], [3], [4]. The state estimation provided by both approaches is given in a form of a set of states at each time instant.

Interval-observer-based approaches are one of the most common approaches in Fault Detection and Isolation (FDI). This approach has appeared in last decade for systems with uncertainties [5], [6], allowing to estimate the state set a timeinstant ahead based on the set estimated in the previous time instant [7]. In fault detection with this family, the parameter uncertainty in non-faulty situation is used for predicting the state [8].

On the other hand, the set-membership state estimation is the other approach for estimating the system state including the measured output and bounded noises [9], [10]. Furthermore, there are several geometrical structures in the literature to compute the set of states in both approaches, e.g., polytopes [11], [12], ellipsoids [13], [14], [15], [16]

\footnotetext{
${ }^{1}$ Supervision, Safety and Automatic Control Research Center (CS2AC) of the Universitat Politècnica de Catalunya, Campus de Terrassa, Gaia Building, Rambla Sant Nebridi, 22, 08222 Terrassa, Barcelona, Spain.

${ }^{2}$ Automatic Control Department of the Universitat Politècnica de Catalunya, Institut de Robòtica i Informàtica Industrial (CSICUPC). Llorens Artigas, 4-6, 08028 Barcelona, Spain. E-mail: \{ mpourasgharlaf, vpuig, cocampo\}eiri.upc.edu
}

and zonotopes [17], [18], [9], [19]. Typically, the set approximation is used to reduce the complexity of computing the uncertain state. Moreover, the set is approximated by means of outer bounds using the outer bounds of the exact uncertain state set at time instant $k-1$ and the measured output set at time instant $k$, where $k \in \mathbb{Z}$.

In order to understand the relation between the intervalobserver-based approach and set-membership approaches, the main objective of this paper is to compare them mathematically and establish mathematical relations between them.

In the present paper, disturbances and noises are the sources of uncertainty considered with unknown magnitudes but known bounds in both state estimation approaches. Also, the discrete-time model is used as a basis for designing state estimation schemes. Moreover, the propagation of uncertainties is done by considering zonotopic representations and associated operations [8].

The main contribution of this paper is to establish the relationship between the observer-based approach and the set-membership approach in the case of single-input, singleoutput (SISO) linear discrete-time systems with bounded uncertain parameters and noise. On the other hand, these relationships are shown by implementing the proposed approaches on the two-tanks case study.

The remainder of the paper is organized as follows. First, some definitions and properties of zonotopes are recalled in Section II. The problem formulation for each approach is studied in Section III. Section IV presents the comparison between the two considered approaches. The mathematical conditions that allow relating both approaches are discussed in Section V. In Section VI, an example based on a twotanks system is proposed in order to show these conditions. Finally, in Section VII conclusions are drown.

\section{THEORETICAL BACKGROUND}

In what follows, some fundamental definitions and properties regarding zonotopes that are important to recall for understanding the contents of this paper are introduced.

Definition 2.1 (Minkowski Sum): The Minkowski sum of two sets is defined by $X \oplus Y=\{x+y: x \in X, y \in Y\}$.

Definition 2.2 (m-order Zonotope): A zonotope is a convex symmetric polytope. Given a vector $p \in \mathbb{R}^{n}$ and a matrix $H \in \mathbb{R}^{n \times m}(n \leq m)$, the zonotope is represented as: $Z=p \oplus$ $H \mathbb{B}^{m}=\left\{p+H z: z \in \mathbb{B}^{m}\right\}$ where $p$ is the center, $H$ contains the segments of the zonotope and $\mathbb{B}^{m}$ is an $m$-dimensional unitary box.

Definition 2.3 (Strip): A strip is described by $S=$ $\left\{x:\left|c^{T} x-d\right| \leq \sigma\right\}$, where $c \in \mathbb{R}$ is a vector and $d, \sigma$ are 
scalars.

Property 2.1: Given two zonotopes $Z_{1}=p_{1} \oplus H \mathbb{B}^{m_{1}} \in$ $\mathbb{R}^{n}$ and $Z_{2}=p_{1} \oplus H \mathbb{B}^{m_{2}} \in \mathbb{R}^{n}$, the Minkowski sum of these zonotopes is defined as $Z=Z_{1} \oplus Z_{2}=\left(p_{1}+p_{2} \oplus\right.$ $\left[\begin{array}{ll}H_{1} & H_{2}\end{array}\right] \mathbb{B}^{m_{1}+m_{2}}$, which is still a zonotope.

Property 2.2: Given the zonotope $Z=p \oplus H \mathbb{B}^{m} \subset \mathbb{R}^{n}$, the strip $S=\left\{x \in \mathbb{R}^{n}:\left|c^{T} x-d\right| \leq \sigma\right\}$ and the vector $\lambda \in \mathbb{R}^{n}$, the intersection between the zonotope and strip is defined as $Z \cap S=\hat{p}(\lambda) \oplus \hat{H}(\lambda) \mathbb{B}^{m+1}$ where $\hat{p}(\lambda)=p+\lambda(d-c p)$ and $\hat{H}(\lambda)=[(I-\lambda c) H \quad \sigma \lambda]$.

\section{PROBLEM FORMULATION}

\section{A. Dynamical Model of the System}

The SISO linear discrete-time invariant system is modeled as

$$
\begin{aligned}
x_{k+1} & =A x_{k}+B u_{k}+\omega_{k}, \\
y_{k} & =C x_{k}+\eta_{k},
\end{aligned}
$$

where $x \in \mathbb{R}^{n}, u \in \mathbb{R}^{p}, y \in \mathbb{R}^{q}$ are the states, inputs and outputs, respectively. Moreover, $A \in \mathbb{R}^{n \times n}, B \in \mathbb{R}^{n \times p}, C \in$ $\mathbb{R}^{q \times n}$ are constant matrices. In (1), $\omega \in W$ denotes the state perturbation (disturbances) and $\eta \in V$ represents the measurement perturbation (noises), both considered as

$$
\begin{gathered}
W=\left\{\omega_{k} \in \mathbb{R}^{r}:\left|\omega_{k}-\omega^{c}\right| \leq \bar{\omega}, \omega^{c} \in \mathbb{R}^{r}, \bar{\omega} \in \mathbb{R}^{r}\right\}, \\
V=\left\{\eta_{k} \in \mathbb{R}^{q}:\left|\eta_{k}-\eta^{c}\right| \leq \bar{\eta}, \eta^{c} \in \mathbb{R}^{q}, \bar{\eta} \in \mathbb{R}^{q}\right\},
\end{gathered}
$$

where $\omega^{c}, \bar{\omega}, v^{c}$ and $\bar{v}$ are constant vectors. Moreover, based on the structures in (2), the zonotopes of $W$ and $V$ can be re-written as

$$
\begin{aligned}
W & =\omega^{c} \oplus H_{\bar{\omega}} \mathbb{B}^{r}, \\
V & =\eta^{c} \oplus H_{\bar{\eta}} \mathbb{B}^{q},
\end{aligned}
$$

where $H_{\bar{\omega}} \in \mathbb{R}^{r \times r}$ and $H_{\bar{\eta}} \in \mathbb{R}^{q \times q}$ are two diagonal matrices with respect to $\bar{\omega}$ and $\bar{\eta}$. Besides, $\mathbb{B}^{r}$ and $\mathbb{B}^{q}$ are unitary boxes.

\section{B. Interval-observer-based Approach}

Based on the dynamical model in (1), the interval observer is used for estimating the state and output sets of the system. The structure of interval observer is expressed as

$$
\begin{aligned}
\hat{x}_{k+1} & =A \hat{x}_{k}+B u_{k}+L\left(y_{k}-\hat{y}_{k}\right)+W, \\
\hat{y}_{k} & =C \hat{x}_{k}+V,
\end{aligned}
$$

where $\hat{x}$ is estimated state, $\hat{y}$ is output and $L$ denotes the observer gain. Moreover, if (1) is assumed to be observable, the observer gain can be designed by using the method proposed in [20] for zonotopic Kalman Filters.

The set definition of the observer structure in (4) can be re-written in the zonotopic form as

$$
\begin{aligned}
\hat{X}_{k+1} & =(A-L C) \hat{X}_{k} \oplus\left\{B u_{k}\right\} \oplus\left\{L y_{k}\right\} \oplus(-L) V \oplus W, \\
\hat{Y}_{k} & =C \hat{X}_{k} \oplus V
\end{aligned}
$$

where $\hat{X}$ and $\hat{Y}$ are the predicted state and output zonotopes, respectively.
Moreover, the zonotopic observer structure in (5) can be propagated in center-segments form as

$$
\begin{aligned}
\hat{x}_{k+1}^{c} & =(A-L C) \hat{x}_{k}^{c}+B u_{k}+L y_{k}-L \eta^{c}+\omega^{c}, \\
\hat{H}_{k+1}^{x} & =\left[\begin{array}{lll}
(A-L C) \hat{H}_{k}^{x} & -L H_{\bar{\eta}} & H_{\bar{\omega}}
\end{array}\right], \\
\hat{y}_{k}^{c} & =C \hat{x}_{k}^{c}+\eta^{c}, \\
\hat{H}_{k}^{y} & =\left[\begin{array}{ll}
C \hat{H}_{k}^{x} & H_{\bar{\eta}}
\end{array}\right],
\end{aligned}
$$

where $\hat{x}_{k+1}^{c}$ and $\hat{H}_{k+1}^{x}$ are the center and segments of $\hat{X}_{k+1}$, respectively. Also, $\hat{y}_{k}^{c}$ and $\hat{H}_{k}^{y}$ are the center and segment of $\hat{Y}_{k}$. In addition, in this computation, the initial zonotopic state is assumed $\hat{X}_{0}=\hat{x}_{0}^{c} \oplus \hat{H}_{0} \mathbb{B}^{m_{0}}$. Moreover, the set of uncertain state can be obtained by using the iterative algorithm that is presented in Figure 1.

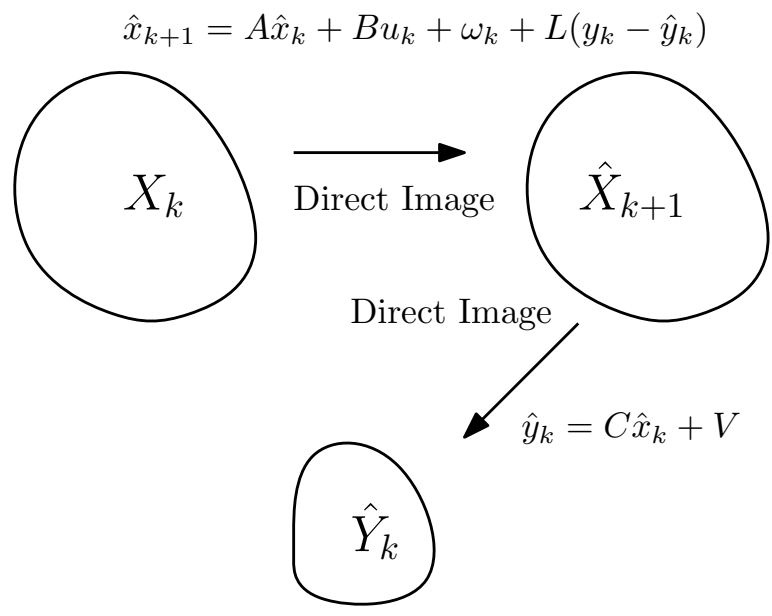

Fig. 1: Iterative algorithm of interval observer approach

\section{Set-membership State Estimation}

Set-membership state estimation is another approach for state estimation. In this approach, the uncertainties are also assumed unknown but bounded.

Based on the above assumption, the uncertain state set can be obtained by using Algorithm 1 (see Figure 2).

\begin{tabular}{l}
\hline Algorithm 1 Set-membership State Estimation \\
\hline 1: Compute the set of predicted states $X_{e}(k)$, \\
2: Compute the set of consistent states $X_{y_{k}}(k)$, \\
3: Compute the set of uncertain states $\hat{X}(k)=X_{e}(k) \cap$ \\
$X_{y_{k}}(k)$.
\end{tabular}

In Algorithm 1, the state estimation is done by means of intersecting the prediction state set $X_{k}^{e}$ and the output strip $X_{k}^{y_{k}}$. This intersection is denoted by $\hat{X}_{k}$.

In addition, the zonotopic representations are used for implementing Algorithm 1 in set-membership approach. In both methods, Step I and II are solved similarly but there are several methods for dealing with Step III (correction step) as singular value decomposition-based method, segments minimization method, volume minimization method and the P-radius minimization method [20]. 


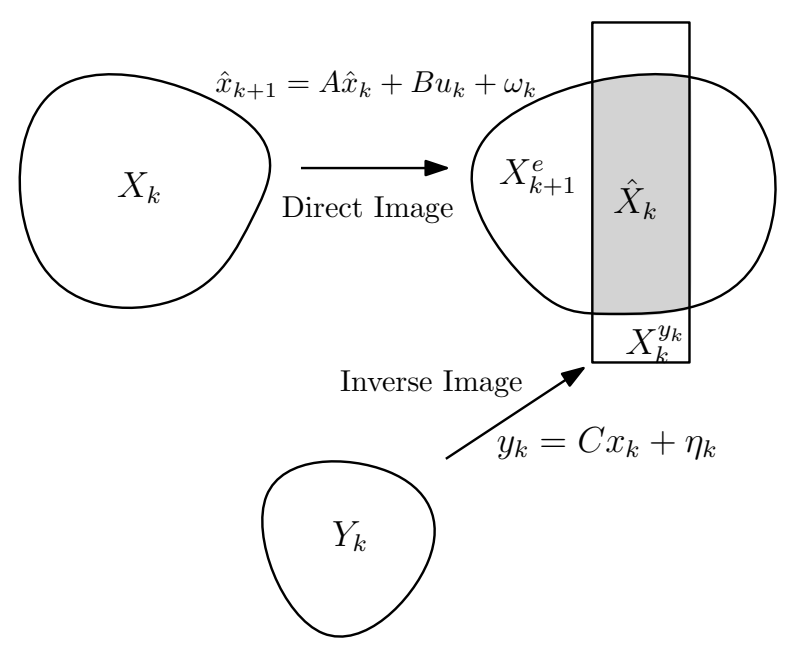

Fig. 2: Set-membership state estimation

Hence, by considering the dynamical model in (1), the Algorithm 1 can be implemented as follows:

- Step I: By considering (1a) and (3a), the prediction state set can be computed as

$$
X_{k+1}^{e}=\hat{x}_{k+1}^{c_{e}} \oplus H_{k+1}^{x_{e}} \mathbb{B}^{r},
$$

where $\hat{x}_{k+1}^{c_{e}}$ and $H_{k+1}^{x_{e}}$ denote the center and the segments of zonotope $X_{k+1}^{e}$, respectively, which are calculated as

$$
\begin{aligned}
\hat{x}_{k+1}^{c_{e}} & =A \hat{x}_{k}+B u_{k}+\omega^{c}, \\
H_{k+1}^{x_{e}} & =\left[\begin{array}{ll}
A \hat{H}_{k}^{x} & H_{\bar{\omega}}
\end{array}\right] .
\end{aligned}
$$

- Step II: The measurements $y_{k}$ are used to obtain the strip in Algorithm 1. This strip can be represented by $S=\left\{x \in \mathbb{R}^{n}:\left|c^{T} x-d\right| \leq \sigma\right\}$, where $d=y_{k}$ and $\sigma$ is the measurement noise. Therefore, the strip can be written as

$$
X_{k}^{y_{k}}=\left\{x \in \mathbb{R}^{n}:\left|C^{T} x-y_{k}\right| \leq \eta_{k}\right\} .
$$

- Step III: The prediction state set and the consistent state set are obtained by considering the dynamical model in (1) in Steps I and II. The intersection between the zonotope obtained in Step I and the Step II gives the state of Step III in Algorithm 1, i.e., $X_{k+1}^{e} \cap X_{k}^{y_{k}}$. Moreover, this intersection can be provided by considering Property 2.2. Therefore, by intersecting the predicted state and the strip in Step III, the state estimation can be obtained in Step III. Hence, based on the prediction step, $X_{k+1}^{e}=\left(A x_{k}+B u_{k}+\omega_{k}\right) \oplus\left[\begin{array}{ll}A \hat{H}_{k}^{x} & H_{\bar{\omega}}\end{array}\right] \mathbb{B}^{r}$, also the strip $X_{k}^{y_{k}}=\left\{x \in \mathbb{R}^{n}:\left|C^{T} x-d\right| \leq \eta_{k}\right\}$, the intersection can be computed as

$$
\hat{X}_{k+1}=\hat{X}_{k+1}^{c}(\lambda) \oplus \hat{H}_{k+1}^{x}(\lambda) \mathbb{B}^{m+1},
$$

where the center $\hat{X}_{k+1}^{c}$ is derived as

$$
\hat{X}_{k+1}^{c}(\lambda)=A \hat{X}_{k}^{c}+\lambda\left(y_{k+1}-C A \hat{X}_{k}^{c}\right)
$$

Also, the segments matrix $\hat{H}_{k+1}^{x}$ is calculated by considering Property 2.2 and (8) as

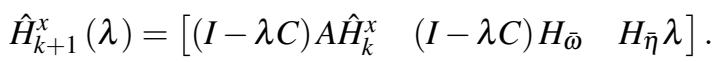

In addition, throughout the procedure of Step III, the whole computation is parameterized by means of vector $\lambda$. Hence, according to [9], it can be generally obtained by $\lambda=\frac{H H^{T} C}{C^{T} H H^{T} C+\sigma^{2}}$ in order to minimize the segments of the zonotope.

\section{COMPARISON BETWEEN BOTH APPROACHES}

The main difference between the interval observer and set-membership approaches is related to their own ways for taking the effect of measurements into the account when performing the state estimation. Interval observer uses an explicit way for achieving this goal through the observer gain. On the other hand, the set-membership approach carries out this process implicitly by providing the intersection between the set of states consistent with the model and the measurements, respectively. Furthermore, the intersection and observer gain that are used in set-membership and interval observer approaches depend on the relative values of process and measurement noises. This fact motivates to find out the relation that can be established between both approaches. Thus, in order to find the mathematical relationship of interval observer and set-membership approaches, the comparison is done by comparing the centers and the segments of the obtained zonotopes.

The predicted center and the segments are computed in (6) with the interval-observer approach. On the other hand, (11) and (12) provide the center and the segments of the zonotopic state estimation with set-membership approach. By comparing these expressions, it is seen that the observer gain and uncertainties play the important role in the interval observer state estimation. On the other hand, the same role is played by the uncertainties in set-membership approach. Therefore, if the same uncertainties are considered for both approaches, the observer gain will be the only important factor for finding the relationship.

In order to find the relation of interval-observer and setmembership approaches, the same disturbance and noise are considered for the dynamical system in (1). The comparison is done under three different conditions according to the observer gain. These conditions are outlined as follows:

- Case I: In this case, the observer gain is selected to satisfy $0<L C<A$. Therefore, the center and the segments of the interval observer can be derived as in (6a) and (6b).

- Case II: In this case, $L=0$ and corresponds to the case that the set of estimated states depends only on the model and disturbances. Then, the zonotope produced by the interval observer can be computed as

$$
\begin{aligned}
\hat{x}_{k+1}^{c} & =A \hat{x}_{k}^{c}+B u_{k}+\omega^{c}, \\
\hat{H}_{k+1}^{x} & =\left[\begin{array}{ll}
A \hat{H}_{k}^{x} & H_{\bar{\omega}}
\end{array}\right] .
\end{aligned}
$$


- Case III: In this case, $L$ is selected to satisfy $L C=A$ (deadbeat observer) such that the set of estimated states depends only on measured inputs and outputs. Thus, the center and the segments of estimated states will be

$$
\begin{gathered}
\hat{x}_{k+1}^{c}=B u_{k}+A \hat{x}_{k}^{c}+\omega^{c}, \\
\hat{H}_{k+1}^{x}=\left[\begin{array}{ll}
-L H_{\bar{\eta}} & H_{\bar{\omega}}
\end{array}\right] .
\end{gathered}
$$

In the first case, the center and the segments are affected by the observer gain. As discussed previously, this gain is obtained by the same method that is proposed in [20], which takes the bounds of uncertainties into account. By comparing (6a) with (11) and (6b) with (12), the centers of both approaches are the same in steady state if the noise will be centered around zero. But, the interval observer set is wider than set-membership approach based on its segments. Additionally, by comparing (13) and (14) for the interval observer with (11) and (12) for set-membership, the state estimations provided by both approaches are affected by disturbances and noises. Moreover, if noise and disturbances are zero mean (that is, centered around zero), for Cases II and III, the centers of interval observer and set-membership approaches will be located in the same place.

On the other hand, by assuming no noise condition, then comparing (13b) with (12) under the same noise condition $\hat{H}_{k+1}^{x}(\lambda)=\left[\begin{array}{ll}(I-\lambda C) A \hat{H}_{k}^{x} & (I-\lambda C) H_{\bar{\omega}}\end{array}\right]$, the only difference between the matrices that are obtained with these two estimation approaches is the optimization vector $\lambda$ from the set-membership approach. It means that, only in the ideal condition $(\lambda=0)$, two approaches will produce the same state predictions.

In the same way for the third case $(L C=A)$, the center of two approaches are the same and (13b) is reduced into $\hat{H}_{k+1}^{x}=\left[H_{\bar{\omega}}\right]$, also in the case without noise. Moreover, it can be concluded that if the center of these approaches are equal, the state that is estimated by interval-observer is inside of the set-membership state estimation.

Summarizing, by supposing the dynamical model under the condition of zero mean noise and in the case of considering that the observer gain satisfies $L=0$ or $L C=A$, the centers of interval observer and set-membership are located in the same positions. Additionally, in the ideal case $\lambda=0$, the approaches are equal if $L=0$ and interval observer is included by set-membership estimation if $L C=A$.

\section{CASE STUDY}

\section{A. Two-Tanks System}

The comparison of the two mentioned state estimator families will be performed by using the two-tanks system that is presented in Figure 3.

The mathematical model of the system can be written as

$$
\begin{aligned}
& \frac{d h_{1}(t)}{d t}=-\frac{a_{1}}{A_{1}} \sqrt{2 g h_{1}(t)}+\frac{a_{2}}{A_{1}} \sqrt{2 g h_{2}(t)}+\frac{\gamma k}{A_{1}} \omega(t), \\
& \frac{d h_{2}(t)}{d t}=-\frac{a_{2}}{A_{2}} \sqrt{2 g h_{2}(t)}+\frac{(1-\gamma) k}{A_{2}} v(t),
\end{aligned}
$$

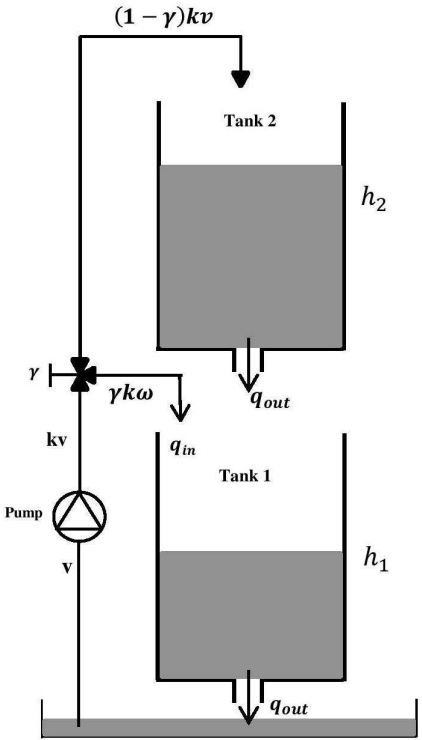

Fig. 3: Schematic diagram of the Two-tanks system

where

- $\gamma$ is the valve ratio.

- $k v(t)$ is the flow through the pump.

- $(1-\gamma) k v(t)$ is the flow towards the Tank 1 according to the valve position.

- $\gamma k \omega(t)$ is the flow towards the Tank 2 according to the valve position.

- $k$ is the pump constant.

- $A_{i}$ is the cross section of Tank $i$, with $i=1,2$.

- $a_{i}$ is the cross sectional area of the outlet pipes.

- $g$ is acceleration due to gravity.

- $h_{i}$ is the level of the water in Tank $i$, with $i=1,2$.

In order to apply the approaches considered in this paper, the previous non-linear model is linearized around the operating point:

- $h_{1}^{*}=12.4[\mathrm{~cm}]$

- $h_{2}^{*}=1.8[\mathrm{~cm}]$,

- $v=3.00[\mathrm{~V}]$,

with the following parameter values:

- $k=3.35\left[\mathrm{~cm}^{3} / \mathrm{Vs}\right]$,

- $\gamma=0.60$.

Including the disturbance and the noise, an Euler discretization (with step size equal to 1 ) of the linearized model can be written based on as [21]

$$
\begin{aligned}
h_{k+1} & =\left[\begin{array}{cc}
-0.0159 & 0.0419 \\
0 & -0.0419
\end{array}\right] h_{k}+\left[\begin{array}{c}
0 \\
0.0479
\end{array}\right] v_{k} \\
& +\left[\begin{array}{c}
0.0718 \\
0
\end{array}\right] \omega_{k}, \\
y_{k} & =\left[\begin{array}{ll}
0.5 & 0
\end{array}\right] h_{k}+\eta_{k},
\end{aligned}
$$

where $\omega$ is the disturbance and $\eta$ is the measurement noise.

Therefore, each condition in Section IV can be checked by using the state-space model in (16) for the case study.

Case I $(0<L C<A)$ : The observer gain in this condition is obtained by using the Zonotopic Kalman Filte method 
$(Z K F)$ that is proposed in [20]. State estimation is done by using interval-observer and set-membership approaches. Figure 4 shows the comparison of estimation with both considered approaches. It can be seen that both approaches present a quite similar behaviour.
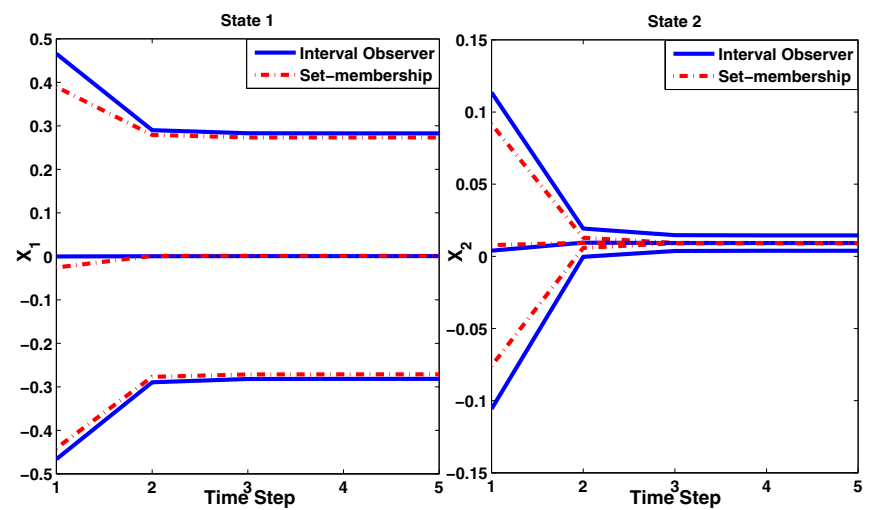

Fig. 4: Envelope of interval observer and set membership state estimation if $0<L C<A$

Moreover, Figure 4 shows, under the condition $0<L C<$ $A$, the bound of state that is estimated by interval observer is wider than set-membership state estimation. This point is shown in Figure 5 by comparing the created zonotopes in the last iteration with both approaches.

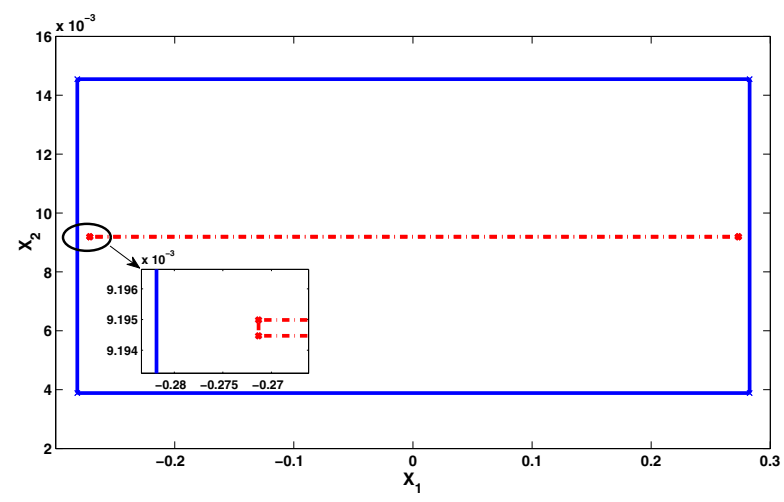

Fig. 5: The zonotope of state estimation in the last iteration by both approaches for $0<L C<A$ (solid-line shows the interval observer and dashed-line presents the set-membership state estimations)

Case II $(L=0)$ : The estimated state is influenced by considering zero value for the gain in interval observer approach. The effect of this gain can be seen in Figure 6 . In this case, the centers and the segments of state estimation in both approaches are converging to each other. Therefore, both approaches have quite similar state estimations.

Moreover, Figure 7 shows that the center are equal and a small difference between the shape of two zonotopes in the last iteration.

Case III $(L C=A)$ : Under this condition, the segment matrix of the state estimation with the interval observer is reduced to one segment that is based on the disturbance. This
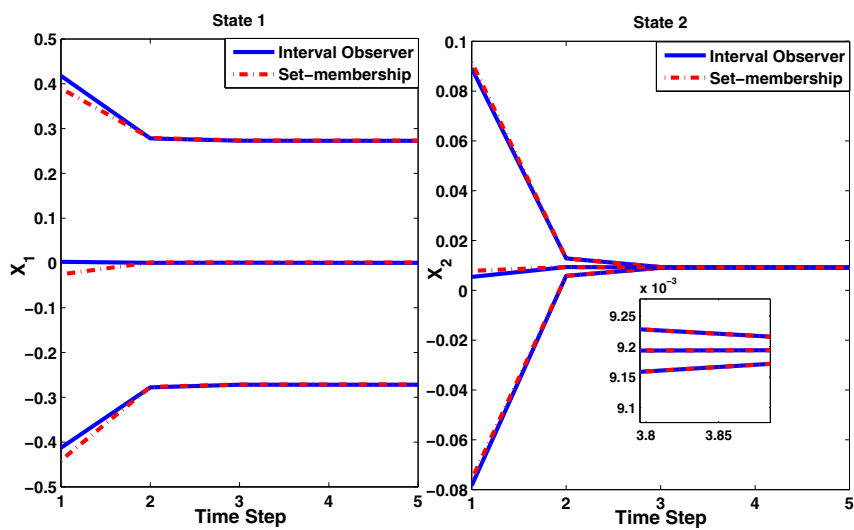

Fig. 6: Envelope of interval observer and set membership when $L=0$

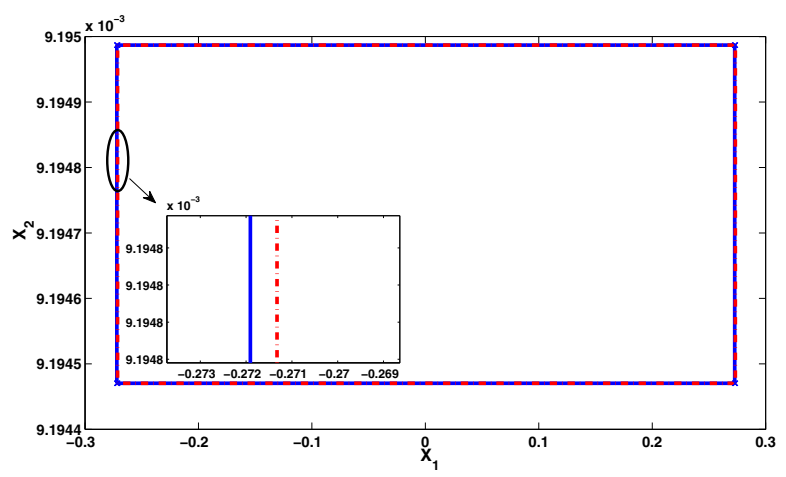

Fig. 7: The zonotope of state estimation in last iteration by both approaches for $L=0$ (solid-line shows the interval observer and dashed-line presents the set-membership state estimations)

disturbance is added to the lower tank during the simulation. Hence, the set of state estimations with interval observer under this condition is obtained as a line.

Figure 8 presents the effect of designing the gain when $L C=A$ on estimating the states. Furthermore, the comparison between two families is shown in this figure. As it is seen, both approaches estimate the similar centers. Therefore, in this case, the set that is estimated by interval observer is inside of the set-membership state estimation, in other words, the interval observer is included by set-membership approach. Figures 8 and 9 show the envelope and last iteration zonotope of both approaches, respectively.

\section{CONCLUSIONS}

The observer-based approach and the set-membership approach have been considered in this paper for estimating the state of linear single-input, single-output (SISO) systems. Moreover, both approaches were analyzed and compared from the mathematical point of view by implementing zonotopes.

In one hand, the calculations involved in both approaches are introduced. On the other hand, by considering the observer gain in interval observer approach, three cases were 

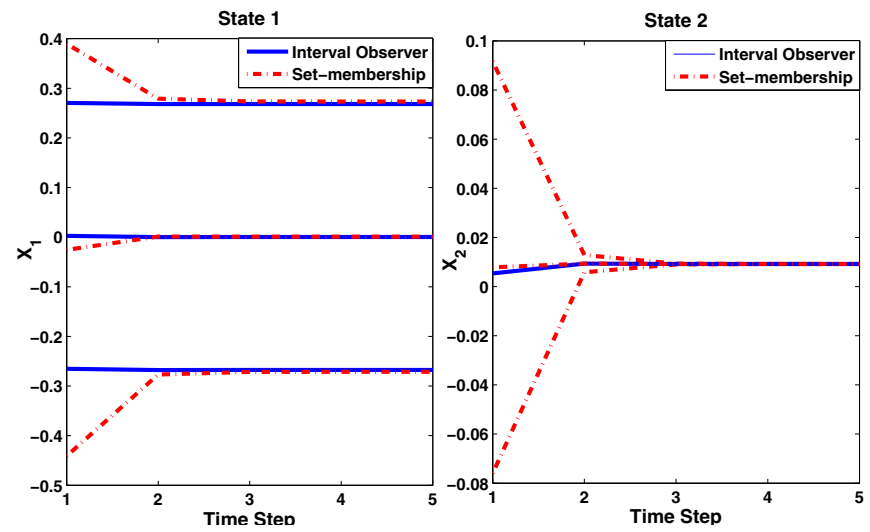

Fig. 8: Envelope of interval observer and set membership if $L C=A$

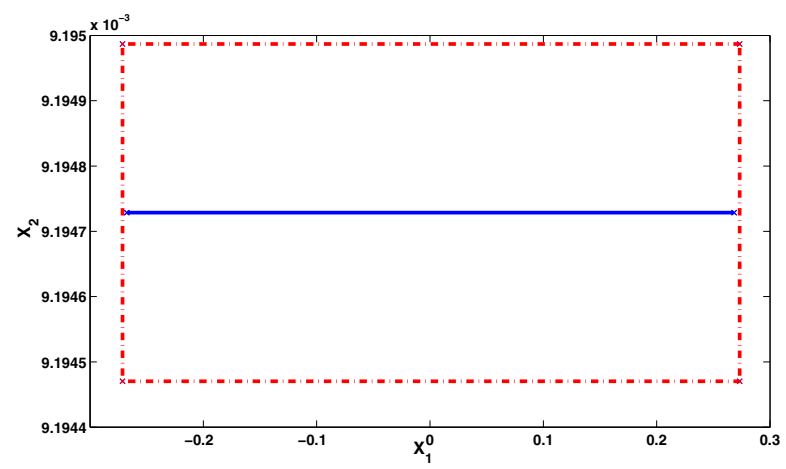

Fig. 9: Envelope of interval observer and set membership if $L C=A$ (solid-line shows the interval observer and dashedline presents the set-membership state estimations)

discussed in order to find the relation between these two families.

In particular, it has been shown that the same envelopes of the state estimation are obtained with both interval observer and set-membership approaches when $L=0$. Furthermore, in the case that the observer gain is designed as $L C=A$ the envelope of state estimation by using interval observer approach goes inside of set-membership envelope. Also, interval observer estimation set included by the one produced by the set-membership if $L C=A$. In the case that the observer gain in the interval observer is designed according to the zonotopic Kalman filter approach, the state estimation envelopes are quite close to those obtained using the setmembership approach. Therefore, it would be one of future research to use some additional conditions for finding the connection of these two families with Kalman filter and Zonotopic Kalman filter state estimators. As a future research, the comparison will be extended to the case of non-linear systems represented in Linear Parameter Varying (LPV) form and to see the performance of both approaches regarding fault detection and isolation.

\section{ACKNOWLEDGEMENTS}

This work has been partially funded by the Spanish Ministry of Science and Technology through the Project
ECOCIS (Ref. DPI2013-48243-C2-1-R).

\section{REFERENCES}

[1] R. E. Kalman. A new approach to linear filtering and prediction problems. Transactions of the ASME-Journal of Basic Engineering, 82(Series D):35-45, 1960.

[2] S. Tornil-Sin, C. Ocampo-Martinez, V. Puig and T. Escobet. Robust fault detection of non-linear systems using set-membership state estimation based on constraint satisfaction. Engineering Applications of Artificial Intelligence, 25(1): 1-10, 2012.

[3] D. Efimov, T. Raïssi, S. Chebotarev, and A. Zolghadri. Interval state observer for nonlinear time varying systems. Automatica, 49(1):200205, 2013.

[4] F. Mazenc and O. Bernard. Interval observers for linear time-invariant systems with disturbances. Automatica, 47(1):140-147, 2011.

[5] Y. Wang, D. M. Bevly and R. Rajamani. Interval observer design for LPV systems with parametric uncertainty. Automatica, 60:79-85, 2015.

[6] T. Raïssi, D. Efimov and A. Zolghadri. Interval state estimation for a class of nonlinear systems. IEEE Transactions on Automatic Control, 57(1):260-265, 2012

[7] F. Mazenc, T. N. Dinh and S. I. Niculescu. Interval observers for discrete time systems. International Journal of Robust and Nonlinear Control, 24(17):2867-2890, 2014.

[8] T. Alamo, J. M. Bravo and E. F. Camacho. Guaranteed state estimation by zonotopes. Automatica, 41(6):1035-1043, 2005.

[9] V. T. H. Le, C. Stoica, T. Alamo, E. F. Camacho and D. Dumur. Zonotope-based set-membership estimation for multi-output uncertain systems. IEEE International Symposium in Intelligent Control (ISIC), pages 212-217, India, 2013.

[10] G. Wei, S. Liu, Y. Song and Y. Liu. Probability-guaranteed setmembership filtering for systems with incomplete measurements. Automatica, 60:12-16, 2015.

[11] R. M. Fernández-Cantí, S. Tornil-Sin, J. Blesa and V. Puig. Nonlinear set-membership identification and fault detection using a Bayesian framework: Application to the wind turbine benchmark. 52nd IEEE Annual Conference in Decision and Control (CDC), pages 496-501, Italy, 2013.

[12] S. M. Tabatabaeipour, P. F. Odgaard, T.Bak and J. Stoustrup. Fault detection of wind turbines with uncertain parameters: a set-membership approach. Energies, 5(7):2424-2448, 2012.

[13] W. Yu, E. Zamora and A. Soria. Ellipsoid SLAM: a novel set membership method for simultaneous localization and mapping. Autonomous Robots, 40(1):125-137, 2016

[14] Z. H. O. U. Bo, Q. I. A. N. Kun, M. A. Xu-Dong and D. A. I. XianZhong. A new nonlinear set membership filter based on guaranteed bounding ellipsoid algorithm. Acta Automatica Sinica, 39(2):146-154, 2013.

[15] K. J. Keesman, J. P. Norton, B. F. Croke, L. T. Newham and A. J. Jakeman. Set-membership approach for identification of parameter and prediction uncertainty in power-law relationships: The case of sediment yield. Environmental modelling and software, 40:171-180, 2013

[16] C. Combastel. A state bounding observer for uncertain non-linear continuous-time systems based on zonotopes. 44th IEEE Conference in Decision and Control, CDC-ECC'05, pages 7228-7234, 2005.

[17] F. Xu, F. Stoican, V. Puig, C. Ocampo-Martinez, and S. Olaru. On the relationship between interval observers and invariant sets in fault detection. In Control and Fault-Tolerant Systems (SysTol), pages 4954, France, 2013.

[18] V. T. H. Le, T. Alamo, E. F. Camacho, C. Stoica and D. Dumur. A new approach for guaranteed state estimation by zonotopes. 18th World Congress IFAC, pages 9242-9247, Italy, 2011.

[19] C. Combastel. A state bounding observer based on zonotopes. Proceedings of European Control Conference, pages 2589-2594, UK, 2003.

[20] C. Combastel. Zonotopes and Kalman observers: Gain optimality under distinct uncertainty paradigms and robust convergence. Automatica, 55:265-273, 2015.

[21] K. H. Johansson. The quadruple-tank process: A multivariable laboratory process with an adjustable zero. IEEE Transactions Control on Systems Technology, 8(3):456-465, 2000. 